\title{
Haltungs- und fütterungsbedingte Erkrankungen des Königspythons
}

Elmar Westenberger

Der Trend zur Haltung exotischer Tiere hält nach wie vor an und dabei steigt die Zahl der Reptilien in privaten Haushalten stetig weiter. Der Königspython ist eine sehr empfindliche Riesenschlange, die aufgrund ihrer vermeintlichen Anspruchslosigkeit häufig ohne ausreichende Grundkenntnisse erworben wird. Dies führt dazu, dass die Tiere regelmäßig mit gesundheitlichen Problemen aufgrund von Haltungs- und Fütterungsfehlern in der Praxis vorgestellt werden.

\section{Der Königspython}

Der Königspython (Python regius) ist die beliebteste und zahlenmäßig am häufigsten gehaltene Riesenschlange. Gründe hierfür sind seine geringe Körpergröße, seine Farbvielfalt, die zahlreichen Zeichnungsmuster, sein umgängliches Verhalten und seine vermeintliche Anspruchslosigkeit. Diese Schlange wird deswegen häufig zu Unrecht als sog. Anfängertier bezeichnet und ohne ausreichende Grundkenntnisse erworben.

In der tierärztlichen Praxis schlägt sich dies in einem erhöhten Aufkommen an Anfragen über die Haltung und Pflege dieser Tiere, in steigenden Patientenzahlen durch haltungsbedingte Erkrankungen und demzufolge in einem steigenden Informationsbedarf nieder. Gleichermaßen steigen die Erwartungen an den Tierarzt hinsichtlich einer fachlich fundierten Behandlung und seines Fachwissens über die Biologie, Pflege und medizinische Versorgung von Reptilien.

\section{Grundsätze für die Haltung}

Für die erfolgreiche Haltung langfristig gesunder Reptilien sind einige grundsätzliche Überlegungen unabdingbar. Alle Reptilien - nicht nur die Wildfänge, sondern auch die Farmzuchten und alle
Tiere, die in menschlicher Obhut geboren wurden oder geschlüpft sind - sind Wildtiere. Ihre Anpassungsfähigkeit an Bedingungen, die von den natürlichen Gegebenheiten abweichen, ist extrem limitiert. Die Konsequenz für die Haltung und Pflege dieser Tiere in menschlicher Obhut ist, dass die Haltungsbedingungen im Terrarium den Bedingungen des natürlichen Lebensraums entsprechen müssen.

\section{.konkret}

Etwa 80 \% aller Erkrankungen bei Reptilien lassen sich auf mangelnde Haltungsbedingungen zurückführen.

Als Wildtiere verfügen Reptilien über ihre ursprünglichen Instinkte. In der freien Wildbahn suchen Fressfeinde stets nach erkennbar kranken und schwachen Tieren. Rangniedere Tiere werden jedes Zeichen von Schwäche bei ranghöheren Artgenossen nutzen, um ihnen den Rang streitig zu machen. Um nicht zur Beute zu werden oder ihren Rang in der artspezifischen Hierarchie zu verlieren, verschleiern erkrankte oder geschwächte Tiere ihr wahres Befinden solange es ihnen möglich ist. Dieses instinktgesteuerte Verhalten führt dazu, dass geringbis mittelgradig schwere Erkrankungszustände für uns als Betrachter nicht ersichtlich sind. Schließlich werden erst im fortgeschrittenen Krankheitsfall äuBerlich Krankheitssymptome sichtbar, die dann häufig sehr unspezifisch sind.

\section{Natürlicher Lebensraum}

Das natürliche Verbreitungsgebiet des Königspythons liegt im feuchtwarmen Klima Westafrikas. Sein Lebensraum sind gras- und buschbewachsene Gebiete mit geringem Baumbestand. Die durchschnittlichen Tagestemperaturen belaufen sich auf $30-35^{\circ} \mathrm{C}$, die mittlere Luftfeuchtigkeit liegt tagsüber bei $70 \%$. Nachts sinken die Temperaturen geringfügig ab, während die Luftfeuchtigkeit zum Morgen hin ansteigt.

\section{Verhalten und Lebensweise}

Als kleine und wenig wehrhafte Riesenschlange ist der Königspython ein sehr sicherheitsbedürftiges Tier, das ein zurückgezogenes Leben führt. Er ist bodenbewohnend und verbringt den Tag überwiegend in dunklen Nagetierbauten oder Termitenhügeln. In diesen Verstecken, die nur einen Eingang aufweisen, herrscht eine konstant hohe Temperatur und Luftfeuchtigkeit. Schlangen sind thigmotaktisch, d.h. der Körperkontakt zu Gegenständen verleiht ihnen Sicherheit ( Abb.1). Deswegen werden von ihnen enge Höhlen bevorzugt, an deren Wänden und Decke sie Kontakt finden.

Hat der Königspython ein seinen Ansprüchen entsprechendes Versteck gefunden, ist er sehr standorttreu. Er verlässt die-

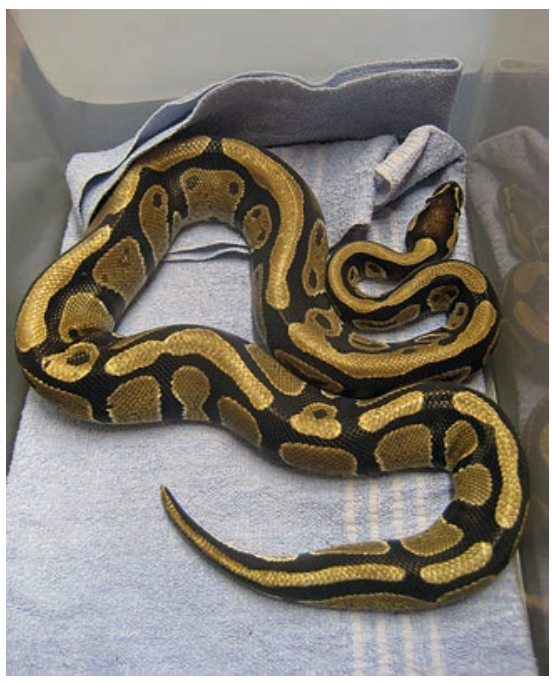

Abb. 1 Selbst während der Untersuchung behält die Schlange Kontakt zur Transportbox. (c) E. Westenberger 
sen Unterschlupf nur zur Nahrungs- oder Partnersuche sowie bei Störungen, die seine Sicherheit beeinträchtigen, z.B. durch Fressfeinde. Den größten Teil seines Lebens verbringt er bewegungsinaktiv im Verborgenen.

Der Python regius ist ein dämmerungsund nachtaktiver Lauerjäger. Seine Hauptaktivitätszeit fällt in die ersten Stunden nach Sonnenuntergang. $\mathrm{Zu}$ seinem Beutespektrum zählen zahlreiche Kleinsäuger und Vögel, wobei der Anteil der erbeuteten Vögel mit zunehmender Körpergröße der Schlange und der damit verbundenen Verminderung der Intensität seiner Kletterbemühungen abnimmt. Darüber hinaus sind Königspythons ausgesprochene Nahrungsspezialisten. So bevorzugt die Riesenschlange innerhalb der Gruppe der adäquaten und verfügbaren Beutetiere häufig individuell nur eine einzige Beutetierart.

\section{Haltung}

\section{Terrarium}

Klassische Vollglasterrarien sind für die Haltung des Königspythons ungeeignet. Neben schlechten energetischen Eigenschaften steht die allseitige Einsehbarkeit im Widerspruch zum Sicherheitsempfinden der Schlange. Zunehmend finden Kunststoffe Verwendung, die sich durch günstige energetische und hygienische Eigenschaften auszeichnen. Darüber hinaus sind diese Terrarien nur von der Vorderseite einsehbar.

Die Mindestgröße des Terrariums ist stets so auszuwählen, dass sie mindestens den Angaben aus dem Gutachten über die Mindestanforderungen an die Haltung von Reptilien von der Sachverständigengruppe tierschutzgerechter Haltung von Terrarientieren des Bundesministeriums für Ernährung und Landwirtschaft (BMEL) entspricht. Danach gelten Riesenschlangen als wenig bewegungsaktive Lauerjäger oder Stöberer, die mögliche Aufenthaltsorte ihrer Beutetiere untersuchen. Ihr Raumbedarf wird somit als gering eingeschätzt.

Die Abmessungen der Haltungseinrichtung sind auf die gesamte Körperlänge der Schlange bezogen. Dabei wird die
Körperlänge jeweils mit einem Faktor für Länge, Breite (Tiefe) und Höhe multipliziert:

- Länge: 1,0 × Körperlänge

- Tiefe: 0,5 × Körperlänge

- Höhe: 0,75 × Körperlänge

Diese Vorgabe gilt für die Unterbringung von maximal 2 Tieren etwa gleicher Größe. Für jedes weitere Tier sind 20\% des Terrarienvolumens unter Beibehaltung der geforderten Terrarienproportionen zuzugeben.

Im Widerspruch dazu steht die häufig praktizierte Haltung der Riesenschlange in tieferen und deutlich niedrigeren Behältnissen. Die Befürworter dieser Haltungsform verweisen auf die überwiegend terrestrische Lebensweise des Königspythons und darauf, dass diese Bedingung dem ausgeprägten Sicherheitsbedürfnis der Riesenschlange eher Rechnung trägt als Terrarien, die den Vorgaben des BMEL entsprechen, also höher als tief sind ( $\triangleright$ Abb. 2 ).

Grundsätzlich sollte die Größe des Terrariums mit der Größe der Schlange mitwachsen. Das Einsetzen eines juvenilen Tieres in ein Terrarium, dessen Größe bereits die Vorgaben für adulte Schlangen erfüllt, ist nicht ratsam. Auch im natürlichen Lebensraum besetzen Jungtiere einen räumlich sehr begrenzten Lebensraum, der exakt ihren Bedürfnissen entspricht. Aufgrund ihrer geringen Körpermasse sind Jungtiere einerseits empfindlicher gegen Auskühlung als adulte Artgenossen, andererseits wärmen sie sich schneller auf. Kleine Terrarien lassen sich exakter klimatisieren als große Terrarien und werden darüber hinaus dem Sicherheitsbedürfnis des juvenilen Königspythons gerecht.

\section{Bodengrund}

Als Bodengrund für ein Terrarium bieten sich unterschiedliche Materialien an. Ein geeigneter Bodengrund hat einen feuchtigkeitsspeichernden Charakter, ohne dauerhaft nass zu sein. Er sollte leicht zu reinigen sein und die Ausscheidungen der Schlange gut aufnehmen. Es eignen sich Materialien, die ein geringes Eigengewicht besitzen und staubfrei sind, z.B.: - Zeitungspapier

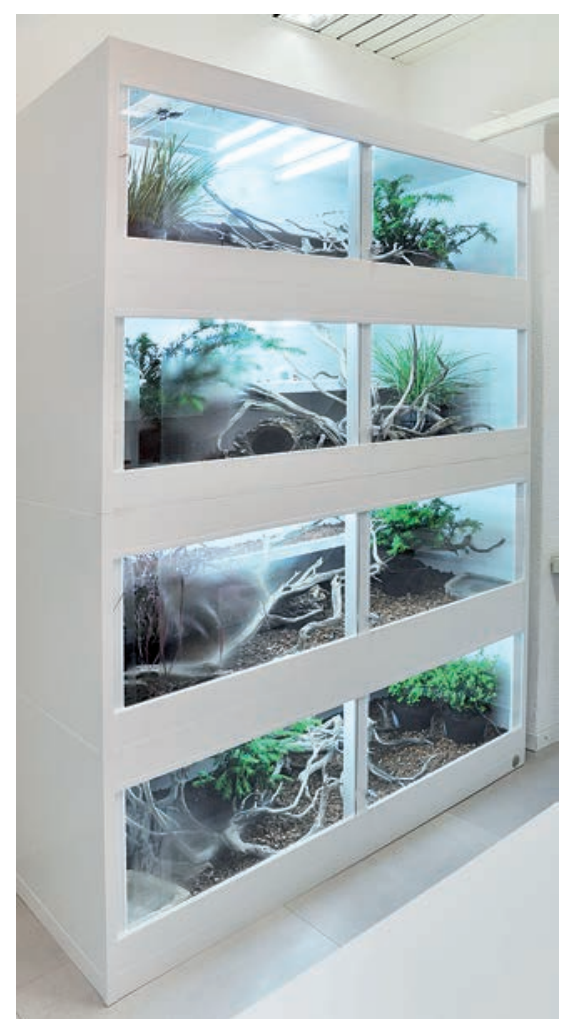

Abb. 2 Vorbildliches Terrariensystem für Königspythons mit naturnaher Einrichtung - obwohl die Höhenvorgabe nicht eingehalten wird. (c) LANZO Herp Cages

- Mulche

- Späne

- spezielle Terrarienerden

- Gartenerde

Da der Königspython keine grabende Schlange ist, ist die Substrathöhe von untergeordneter Bedeutung.

\section{Standort des Terrariums}

Als Standort des Terrariums sollte ein möglichst ruhiger und störungsfreier Platz gewählt werden. Ein unruhiger Standort in belebter, häuslicher Umgebung und starke Schwingungen (z.B. durch Musik oder den Verkehr an vielbefahrenen Straßen) führen zu einer andauernden Stressbelastung mit negativen Folgen für die Tiere. Optimal sind separate Zimmer, in denen ausschließlich Reptilien gepflegt werden.

In diesen Räumen sollte die Mindesttemperatur immer der Mindesttemperatur entsprechen, die die Terrarienbewohner bevorzugen, da es nahezu unmöglich ist, den verhältnismäßig kleinen Terrarienraum unabhängig von den Tem- 
peraturgegebenheiten des Zimmerraums zu klimatisieren. Beim Königspython beträgt die nächtliche Mindesttemperatur $23^{\circ} \mathrm{C}$. Das Klima im Terrarium darf nicht durch äußere Wärmequellen, z.B. durch direkte Sonneneinstrahlung durch ein Fenster, beeinträchtigt werden, andernfalls besteht Überhitzungsgefahr.

\section{Gruppenzusammensetzung}

Schlangen sind Einzelgänger, die sich nur zur Paarungszeit auf die Suche nach einem Geschlechtspartner begeben. Der Königspython besitzt ein sehr geringes intraspezifisches Aggressionspotenzial. Leider wird dies häufig in der Art und Weise missverstanden, dass sich mehrere Schlangen ein Terrarium teilen müssen. Die dauernde Vergesellschaftung mit Artgenossen führt jedoch zu einer dauerhaften Stressbelastung der Tiere mit negativen gesundheitlichen Folgen.

\section{.konkret}

Königspythons sind Einzelgänger und sollten nicht in einer Gruppe gehalten werden.

\section{Klima}

Die klimatischen Bedingungen im Terrarium variieren in Abhängigkeit von $\mathrm{Be}$ leuchtung, Temperatur und Luftfeuchtigkeit.

\section{Beleuchtung}

Unter dem Oberbegriff Beleuchtung werden die Lichtstärke, die Art der Lichtwellenlängen und die Beleuchtungsdauer zusammengefasst. Grundsätzlich

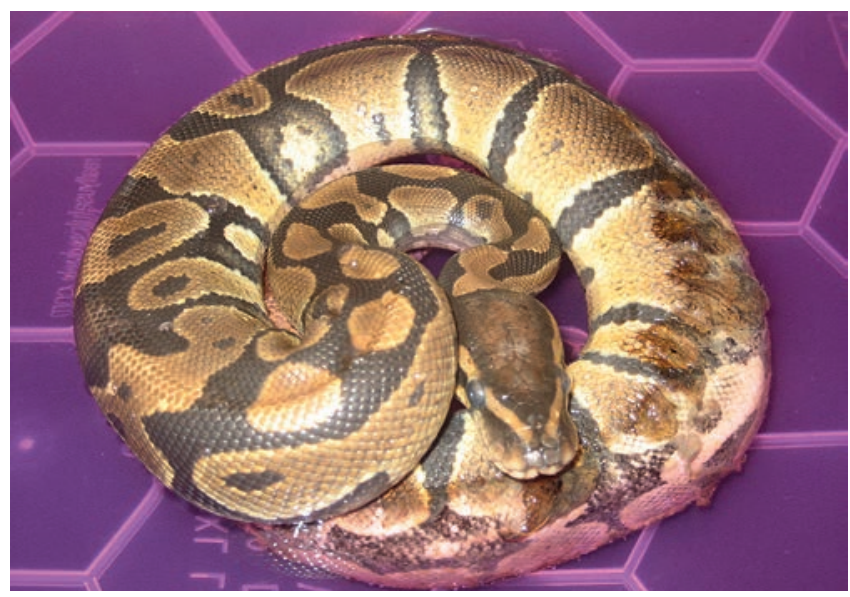

Abb. 3

Königspython mit Hautverbrennungen während der Badebehandlung in Kaliumpermanganatlösung.

(C) E. Westenberger
Unter natürlichen Bedingungen ist das Sonnenlicht immer mit Wärmestrahlung verbunden, daher ist eine Wärmequelle von unten unphysiologisch. Deswegen sollten im Terrarium Licht und Wärme immer verknüpft werden, z.B mit Spotstrahlern. Die Strahler dürfen für die Tiere nicht erreichbar sein und müssen ggf. mit einem Gitterkäfig ummantelt werden. Da Reptilien keine lokale Überhitzung registrieren können, sondern nur eine vollständige Überhitzung des gesamten Körpers, kann es bei Berührung mit heißen Gegenständen zu erheblichen Verbrennungen kommen ( $\triangleright$ Abb.3). Heizmatten oder -kabel sollten nur von außen an den Seitenwänden des Terrariums angebracht werden, um eine gewisse Grundwärme zu erreichen. Die Tiere sollten nie die Möglichkeit haben, sich unter eine Heizmatte zu „graben“. Durch die entstehende Stauwärme kommt es sonst zum Hitzeschock und zu schweren Verbrennungen.

Bezüglich der Temperaturen und Luftfeuchte muss im Terrarium für Königspythons zwischen 2 Klimabereichen unterschieden werden:

- dem Klima im Versteck, in dem der Königspython den größten Teil seines Lebens verbringt

- dem Klima im übrigen Terrarienraum während der Nacht, wenn der Königspython sein Versteck verlässt

Die Vorzugstemperatur im Versteck soll dauerhaft und ohne Schwankungen bei $31-32^{\circ} \mathrm{C}$ liegen bei einer durchgehend hohen Luftfeuchtigkeit von ca. $80 \%$. Im übrigen Terrarium muss tagsüber ein horizontaler Temperaturgradient hergestellt werden, der zwischen 26 und $33^{\circ} \mathrm{C}$ liegt. Nachts wird die Temperatur auf $23^{\circ} \mathrm{C}$ abgesenkt. Die Luftfeuchtigkeit liegt tagsüber vorzugsweise bei über $60 \%$ und steigt nachts noch an.

\section{Strukturierung des Terrariums}

Das wichtigste Strukturelement des Terrariums ist der Versteckplatz. In Anlehnung an die natürlichen Gegebenheiten soll dieser nur einen Zugang besitzen ( Abb.4). Die Größe des Verstecks muss so gewählt werden, dass die Schlange in eingeschlungenem Zustand an den Wänden und der Decke des Unterschlupfs 


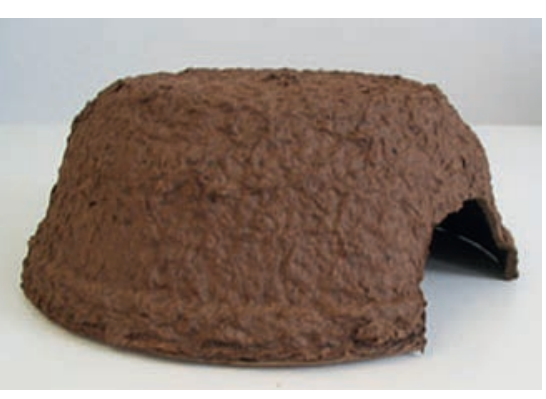

Abb. 4 Unterschlupf aus Zellstoff mit einem Zugang. Dieses Material speichert Feuchtigkeit und gewährleistet ein dauerhaft feuchtes Innenklima. (c) LANZO Herp Cages

Kontakt findet. Weiterhin ist ein Trinkgefäß unerlässlich. Die Strukturierung des restlichen Terrarienraums kann sehr variabel und entsprechend den optischen Wünschen des Betrachters gestaltet werden. Durch den Einbau weiterer Ebenen lässt sich die den Schlangen zur Verfügung stehende Fläche vergrößern.

\section{Fütterung}

Schlangen sind ausschließlich carnivor und werden mit lebenden oder toten Futtertieren gefüttert. Zur Verminderung von Stress für das Futtertier und zur Minimierung von Gefahren für die Schlange ist die Verfütterung toter Futtertiere stets zu bevorzugen. Im Gegensatz zur Fütterung von herbivoren oder insektivoren Reptilien, bei denen auf fütterungsbedingte Mangelzustände ein großes Augenmerk gelegt werden muss, ist die Fütterung ganzer Futtertiere bei Schlangen stets bedarfsdeckend, z.B. hinsichtlich der Versorgung mit Kalzium. Sind die Futtertiere ihrerseits ausgewogen ernährt worden und werden mit einem gefüllten Magen-Darm-Trakt verfüttert, sind ernährungsbedingte Erkrankungen bei Schlangen selten.

Zur Fütterung des Königspythons eignen sich diverse Kleinnagerarten verschiedener Altersstufen. Die Größe der angebotenen Futtertiere und die Frequenz der Fütterung richten sich nach dem Alter und der Größe der Schlange. Folgende Fütterungsintervalle sind empfehlenswert:

- Jungtiere bis zu 6 Monate: alle 5-7 Tage

- Halbwüchsige: alle 10-14 Tage
- Adulte ab einer Körperlänge von ca. $90 \mathrm{~cm}$ : alle 14-21 Tage

\section{.konkret}

Während der Häutungsphase werden Königspythons nicht gefüttert.

Wenn - entgegen der Empfehlung mehrere Tiere gemeinsam gehalten werden, sollten sie stets einzeln und außerhalb des Terrariums in kleinen Einzelboxen gefüttert werden, um Streitigkeiten um das Futter zu vermeiden. Außerdem sind Schlangen, die außerhalb ihres Terrariums gefüttert werden, bei Pflegearbeiten im Terrarium umgänglicher, weil sie an diesem Ort kein Futter erwarten.

\section{Leitsymptome bei Reptilien}

Bei Reptilien sind Symptome, die für eine spezifische Krankheit kennzeichnend sind, sehr selten. In der Regel werden nur allgemeine Symptome sichtbar, die dann hinsichtlich ihrer Krankheitsursachen differenzialdiagnostisch aufgearbeitet werden müssen. Zu den häufigsten Leitsymtomen bei Schlangen zählen Inappetenz, Erbrechen und Häutungsstörungen.

\section{Inappetenz}

Das Symptom Inappetenz ist sehr unspezifisch. Die Futterverweigerung kann bei Schlangen physiologische und pathologische Ursachen haben.

\section{Physiologische Ursachen für Inappetenz}

$\mathrm{Zu}$ den physiologischen Ursachen gehören:

- Häutung

- Trächtigkeit

- Paarungszeit

- Eingewöhnungszeit

- Sättigung

- Fresspausen

\section{Häutung}

Kurz vor der Häutung nehmen die meisten Schlangen keine Nahrung auf. Die Häutungsphase nimmt einen Zeitraum von 1-2 Wochen ein. Die bevorstehende Häutung ist optisch an der Trübung der Brille erkennbar ( $\mathbf{A b b} \mathbf{6}$ ), die jedoch

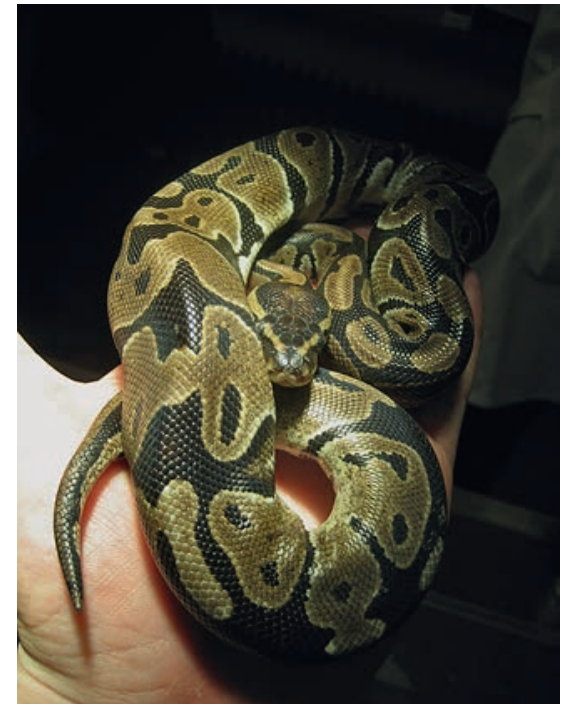

Abb. 5 Der Königspython ist eine sehr umgängliche Riesenschlange. Trotzdem führt ein zu häufiges Handling zu Stress. (C) E. Westenberger

unmittelbar vor der Häutung wieder verschwindet. Zudem trübt sich die Farbe der Haut, weil sich Flüssigkeit zwischen alter und neuer Hautschicht einlagert.

Die 1. Häutung findet meist 1 Woche nach dem Schlupf statt. Die Jungtiere nehmen zuvor grundsätzlich kein Futter, sondern nur die Nahrungsreserven des Dottersacks auf.

\section{Trächtigkeit}

Weibliche Schlangen stellen gewöhnlich 8-3 Wochen vor der Eiablage die Nahrungsaufnahme ein und beginnen mit der Suche nach einem geeigneten Eiablageplatz. Die Trächtigkeitsdauer liegt bei 120-130 Tagen. Beim Königspython ist ein „auf die Seite-Legen“ des hinteren Körperdrittels ein typisches Zeichen für eine fortgeschrittene Trächtigkeit.

\section{Paarungszeit}

Männliche Schlangen verweigern die Nahrungsaufnahme regelmäßig während der Paarungszeit.

\section{Eingewöhnungszeit}

Neuerworbene Schlangen zeigen in der Eingewöhnungszeit oder direkt nach dem Umsetzen in ein neues Terrarium eine temporäre Inappetenz. Abhängig vom Charakter der Riesenschlange kann dieser Zeitraum stark variieren. Regelmäßige Bestimmungen des Körperge- 


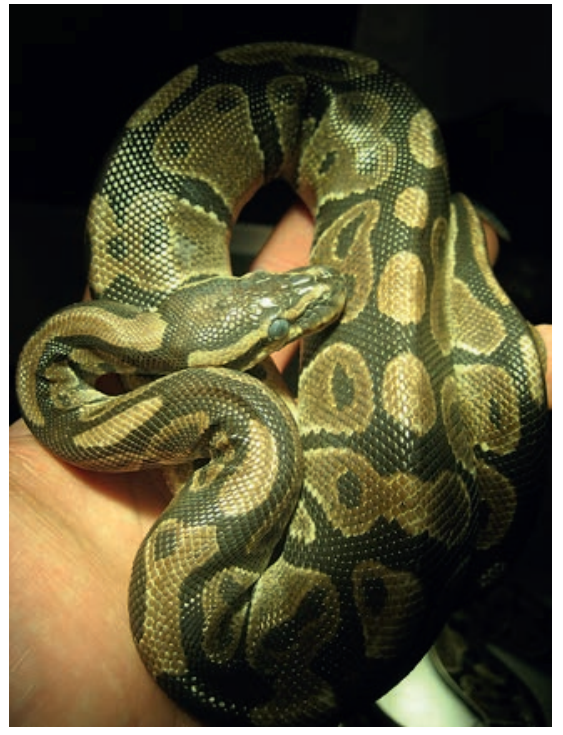

Abb. 6 Königspython vor der Häutung mit deutlicher Trübung der Brille. Der Begriff Brille bezeichnet die verwachsenen und durchsichtig gewordenen Augenlider von Schlangen. Die Brille häutet sich mit. (c) E. Westenberger

wichts sind bei der Einschätzung eines Therapiebedarfs hilfreich. Bei allgemein gesunden Tieren verändert sich das Körpergewicht in diesen Phasen nicht.

\section{Sättigung}

Ein weiterer physiologischer Grund für Inappetenz ist die Sättigung. Während des Verdauungsvorgangs, der Tage bis Wochen in Anspruch nehmen kann, nehmen Schlangen keine neue Nahrung auf.

\section{Fresspausen}

Neben diesen physiologischen Gegebenheiten, die für alle Schlangen Geltung finden, legt insbesondere der Königspython - im natürlichen Lebensraum in der trockenen Jahreszeit wie auch im Terrarium - physiologischerweise lange Fresspausen ein. Diese können sich über einen Zeitraum von mehreren Monaten erstrecken. Während dieser Zeit verlieren die Schlangen jedoch keine bzw. kaum an Körpermasse.

\section{Pathologische Ursachen für Inappetenz}

Eine pathologische Inappetenz kann auf Fehlern in der Haltung und Fütterung beruhen. Die Gründe können nur im Rahmen einer ausführlichen Anamnese ermittelt werden: Am besten sollte der Tierhalter vor der Konsultation einen detaillierten Anamnesebogen mit allen relevanten Fragen zur Haltung und Fütterung ausfüllen.

\section{Stress}

Der Königspython ist äußerst sensibel und stressanfällig. Das Fehlen eines adäquaten Versteckplatzes, ein unruhiger Standort des Terrariums, die Haltung mehrerer Tiere in einem Terrarium oder ein zu häufiges Handling ( $\triangleright$ Abb.5) stellen Stressfaktoren dar. Die übliche Antwort auf derartige Stressoren ist die Nahrungsverweigerung. Bei Wildfängen stellen Fang, Hälterung beim Zwischenhändler in Afrika, Transporte nach Europa und eine erneute Zwischenhälterung beim Zoohändler eine massive Stressbelastung dar, die oftmals mit monatelanger Futterverweigerung beantwortet wird.

\section{Futtertiere}

Königspythons gelten als Nahrungsspezialisten. Insbesondere Wildfänge oder Tiere aus Ranchingfarmen sind oft auf eine spezielle Futtertierart, sogar auf eine spezielle Fellfarbe geprägt. Werden diesen Tieren andere als die bekannten Futtertiere angeboten, verweigern sie konsequent die Nahrungsaufnahme. Bei inappetenten Tieren mit verdächtiger Herkunft kann die ausschließliche Verfütterung von Rennmäusen (Gerbils) die Lösung des Problems sein.

Ein weiterer Grund für die Futterverweigerung kann die Prägung auf tote oder lebende Beute sein. Eine Schlange, die stets mit lebender Beute gefüttert wurde, kann tote Beutetiere verweigern und umgekehrt. Auch die Größe der Beutetiere kann Anlass zur Futterverweigerung geben. Während zu kleine Futtertiere häufig nicht beachtet werden, können Königspythons aus Respekt vor zu großen Futtertieren deren Aufnahme verweigern. Darüber hinaus können wehrhafte Futtertiere wie Ratten, Vielzitzenmäuse und Rennmäuse zu Stresssituationen führen. Wird ein Königspython von einem Beutetier gebissen, kann er eine Vorsicht gegenüber dieser Futtertierart entwickeln und diese als Futter oder sogar komplett die Nahrungsaufnahme verweigern. Solchen Schlangen sollte man viel Ruhe gönnen und ihnen zunächst weniger wehrhafte Futtertiere (Mäuse) anbieten.

\section{Fütterungszeit}

Als dämmerungs- und nachtaktive Riesenschlange werden Königspythons stets am Abend gefüttert. Wird eine andere Fütterungszeit gewählt, verweigern die Tiere die Nahrungsaufnahme.

\section{Überfütterung}

Sind Schlangen durch Überfütterung stark verfettet, legen sie nicht selten monatelange Fresspausen ein.

\section{Haltungstemperaturen}

Als wechselwarme Tiere benötigen die Riesenschlangen Klimabedingungen, mit denen sie ihre bevorzugte Körpertemperatur (preferred Body Temperature, PBT) erreichen können. $\mathrm{Zu}$ niedrige Temperaturen führen dazu, dass der Stoffwechsel suboptimal abläuft. Dies äußert sich u.a. in Futterverweigerung.

\section{Erkrankungen}

Die Nahrungsverweigerung kann beim Königspython zusätzlich zu den haltungsbedingten Gründen auch ein klinisches Symptom für Erkrankungen sein (z.B. Infektionen mit Parasiten, Bakterien, Viren sowie Organerkrankungen). Sollten sich hierfür Verdachtsmomente ergeben, muss eine weitere Diagnostik erfolgen. An die klinische Allgemeinuntersuchung schließen sich in der Regel Blut- und Kotuntersuchungen sowie eine bildgebende Diagnostik an.

\section{Erbrechen}

Die häufigsten Gründe für das Erbrechen sind Haltungsfehler und parasitäre, virale oder bakterielle Infektionskrankheiten. Selten sind Fremdkörper, Abszesse oder Neoplasien in Ösophagus oder Magen ursächlich. Da es sich grundsätzlich um ein unspezifisches Symptom handelt, ist eine sorgfältige Anamnese der Haltungsbedingungen unabdingbar.

\section{.konkret}

Das Leitsymptom Erbrechen wird stets von pathologischen Ursachen hervorgerufen. 


\section{Stress}

$\mathrm{Zu}$ den wichtigsten haltungsbedingten Gründen für Erbrechen bei Schlangen zählt Stress. Die Tiere können z.B. erbrechen, wenn im Terrarium keine geeigneten Versteckplätze zur Verfügung stehen, oder wenn sie kurz nach der Fütterung z.B. durch Reinigungsarbeiten - aus dem Terrarium genommen oder transportiert werden. Eine hohe Besatzdichte und eine Infektion mit Ornithonyssus natricis (der Schlangenmilbe) können ebenfalls zu stressbedingtem Erbrechen führen.

\section{Niedrige Umgebungstemperaturen}

Die Haltung bei niedrigen Umgebungstemperaturen, unter denen die artspezifische, bevorzugte Körpertemperatur (PBT) nicht erreicht werden kann, führt zu einer Minderfunktion der zur Verdauung notwendigen Enzymaktivitäten. Unter unzureichenden Verdauungsbedingungen können die Beutetiere zunehmend in Verwesung übergehen und werden erbrochen.

\section{Infektionen}

Eine Kryptosporidieninfektion verursacht bei Schlangen eine Hypertrophie der Magenwand. Der Magen wird dann schon bei äußerlicher Betrachtung des Tieres als Verdickung am Übergang vom 1. zum 2. Körperdrittel sichtbar. Betroffene Tiere erbrechen ihr Futter in der Regel 1-3 Tage nach der Nahrungsaufnahme. Zudem gehen Infektionen mit Flagellaten, Amöben, Nematoden oder Zestoden mit dem Symptom Erbrechen einher.

Schließlich sind auch bakterielle und virale Infektionen in Betracht zu ziehen. Der Verdauungstrakt von Schlangen wird physiologischerweise von zahlreichen Bakterienarten besiedelt. Im Erkrankungsfall verändert sich die zahlenmäßige Verteilung der Bakterien hin zu pathogenen Arten, deren Wachstum oft durch abwehrschwächende Einflüsse fehlerhafter Haltung - begünstigt wird. In bakteriologischen Untersuchungen zeigt sich dies, indem die physiologische, bakterielle Mischflora durch pathogene Erreger in Reinkultur ersetzt wird.

\section{Håutungsschwierigkeiten}

Wie alle Reptilien wächst auch der Königspython lebenslang. Die Wachstums- geschwindigkeit verlangsamt sich mit zunehmendem Alter jedoch so sehr, dass die Größenzunahme optisch nicht mehr erkennbar ist. Da die äußeren Hautschichten nicht mitwachsen, werden sie im Rahmen des Häutungsvorgangs abgestoßen. Die Frequenz der Häutungen nimmt mit zunehmendem Alter ab: Junge Königspythons häuten sich im 4bis 6-wöchigen Rhythmus, adulte Tiere nur noch wenige Male im Jahr.

Vor der Häutung lagert sich Flüssigkeit zwischen der abzustoßenden und der nachgewachsenen Hautschicht ein. Dies wird durch die Eintrübung der Brille und der milchig hellen Verfärbung der gesamten Haut für einen Zeitraum von 4-7 Tagen sichtbar ( $\triangleright$ Abb.6). Die Häutungsflüssigkeit wird 2-4 Tage vor der Häutung resorbiert. Die Trübung verschwindet und die Schlange erlangt ihre Färbung zurück. Durch Reiben an rauen Gegenständen öffnet die Schlange die Haut im Kopfbereich und streift sie in einem Stück, dem sog. Natternhemd, ab.

\section{Häutungsstörungen}

Häufige haltungsbedingte Ursache für Häutungsstörungen sind die Haltung unter zu trockenen oder zu kühlen Klimabedingungen und das Fehlen von adäquaten Häutungshilfen (Steine, Äste oder andere raue Gegenstände, an denen sich die Schlange reiben kann). Weiterhin können ein allgemein schlechter Gesundheitszustand, Ektoparasitenbefall, Infektionen oder Narbenbildung dazu führen, dass sich Schlangen nur unvollständig häuten. Bei zu feuchten Haltungsbedingungen, unter denen der Boden im Terrarium nicht abtrocknet, kommt es häufig zu Mykosen an den Bauchschuppen. Weiterhin kann eine Flüssigkeitsansammlung unter bzw. in den ventralen Schuppen zu einer Bläschenbildung führen. Bakterielle Infektionen der so vorgeschädigten Haut führen zu schweren Dermatitiden.

\section{Weitere haltungbedingte Vorstellungsgrüinde}

Sämtliche Stoffwechselvorgänge, insbesondere die Immunabwehr, sind bei Reptilien temperaturabhängig. Die Tiere sind darauf angewiesen, ihre Körpertempera-

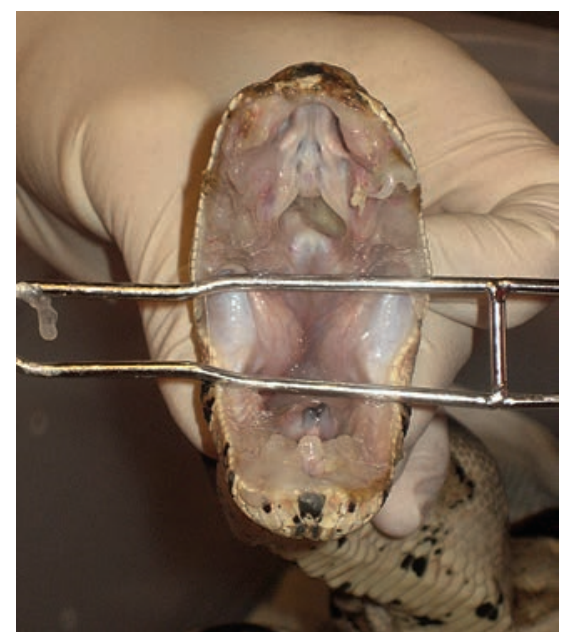

Abb. 7 Stomatitis und deutliche Schleimansammlung infolge einer Infektion der Atemwege. @ E. Westenberger

tur möglichst lange im Bereich ihrer artspezifisch bevorzugten Körperinnentemperatur zu halten. Unter suboptimalen, zu kühlen Haltungsbedingungen nimmt die Leistungsfähigkeit der körpereigenen Immunabwehr ab und die Anfälligkeit gegenüber Infektionserregern zu. Es treten gehäuft Atemwegsinfektionen und Stomatitiden auf ( Abb. 7).

\section{Fazit}

Der Königspython ist eine sehr empfindliche Riesenschlange. Regelmäßig führen Fehler in der Haltung und Fütterung zu gesundheitlichen Problemen. Um diesen vorzubeugen, sind spezifische Kenntnisse über den natürlichen Lebensraum und die spezielle Lebensweise der Schlange essenziell. Haltungsbedingte krankheitsbegünstigende oder auslösende Faktoren müssen gezielt vor der tierärztlichen Untersuchung mittels eines sehr detaillierten Vorberichts erkannt und bewertet oder ausgeschlossen werden.

Online zu finden unter

http://dx.doi.org/10.1055/s-0035-1547400

\section{Dr. Elmar Westenberger}

Zusatzbezeichnung Reptilien

Tierklinik Dr. W. Doering \& Partner

Umbachsweg 8

34123 Kassel

info@tierarzt-doering.de 